\title{
Transmission Spectrum Shifting By Varying Ambient Refractive Index in LPFG
}

\author{
Furat.y.Abdul-Razak \\ Furat_al2006@yahoo.com \\ Dept.of Software Eng., University of Mosul,Iraq
}

\begin{abstract}
In this paper,we introduce a new model, of long-period fiber grating, by taking the value of ambient refractive index greater than the refractive cladding index, where the difference between them must equal $(\approx 0.2)$. The results show the change in power attenuation coefficient, where it increases with the increase in refractive ambient index. The power attenuation coefficient shift shows a dramatic change of a sharp increase from $0.00 \mathrm{~dB}$ to $\approx 0.03788 \mathrm{~dB}$ and $0.00 \mathrm{~dB}$ to $\approx 0.04675 \mathrm{~dB}$ and $0.00 \mathrm{~dB}$ to $\approx 0.06875 \mathrm{~dB}$. Here this model is used as sensor for shifting in input signal $(15 \mathrm{~nm}, 5 \mathrm{~nm})$.
\end{abstract}

Key Word: Long Period Fiber Grating, single mode fiber optical.

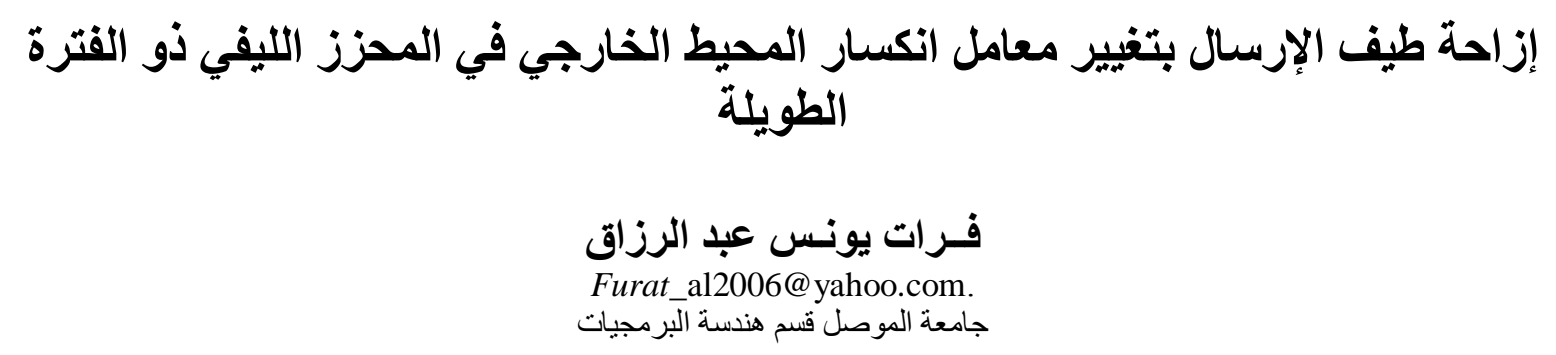

\footnotetext{
الخلاصـة

في هذا البحث قدمنا نموذجآ جديدآ من المحزز الليفي ذو الفترة الطويلة, من خلال اخذ قيمة معامل الانكسار للمحيط

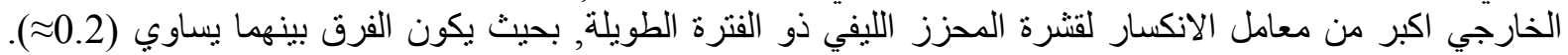

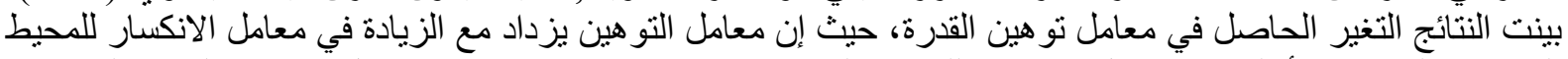

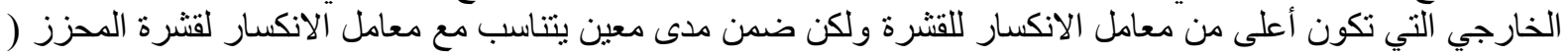
يزداد من استخدام هذا النموذج كمتحس للاز احة في أثنارة الإدخال (15nm, 5nm).
} 


\section{1-Introduction:}

Fiber Optic Gratings or Fiber Bragg Gratings (FBG) were first reported in 1978 by Hill et al. However, such devices only attracted the researcher's attention in 1989, when new production techniques allowed their use with optical communication wavelengths. Several methods exist for FBG production, among them the direct phase mask writing and phase mask interferometers stand out [1,2].

A new class of fiber grating called Long Period Fiber Grating (LPFG) was demonstrated by Vengsarkar et al in 1996.The name is due to the refractive index change periodicity from $100 \mu \mathrm{m}$ to $700 \mu \mathrm{m}$, about 100 times larger than the values employed for FBG formation. This difference makes possible the use of amplitude masks instead of phase masks, resulting in lower manufacturing costs when compared to the FBG production's costs. Besides this, the LPFG presents other surpassing characteristics such as low insertion losses, low back reflection, relatively simple fabrication, and a high sensitivity to changes in physical external parameters. These features made the LPFG outstanding devices for application such as band rejection filters and gain equalizing filters in optical communication, beyond its wide applicability as a fiber optic sensor.

A long-period fiber grating, which couples light from fundamental guided core mode into co-propagating cladding modes at various wavelengths. The LPFG have also been used as gain-flattening filter and as optical fiber polarizer. Unlike FBG the cladding mode configuration of the LPFG is extremely sensitive to the refractive index of the medium surrounding the cladding, thus allowing it to be used as an ambient index sensor. The wavelength at which the coupling from core to cladding modes takes place is directly dependent on the difference between the core and cladding indices, the dimensions of the core and cladding and grating period, and any change in these values can shift the transmission spectral profile [1,2, and 3].

\section{2-Long-Period Fiber Grating(LPFG):}

Long period gratings are fiber optics based devices made up of periodic changes in core's refractive index [2]. Photo induced long-period fiber gratings (LPFG) with periods $L_{L P G}=10^{2}-10^{3} \mu \mathrm{m}$. LPG is transmission grating in which the coupling is between forward propagation core and cladding modes, propagation in the same direction. Typically in a single mode fiber (see figure 1) an LPFG couples the fundamental guided core mode to a copropagating cladding mode at a coupling (or resonance) wavelength $[1,2]$.

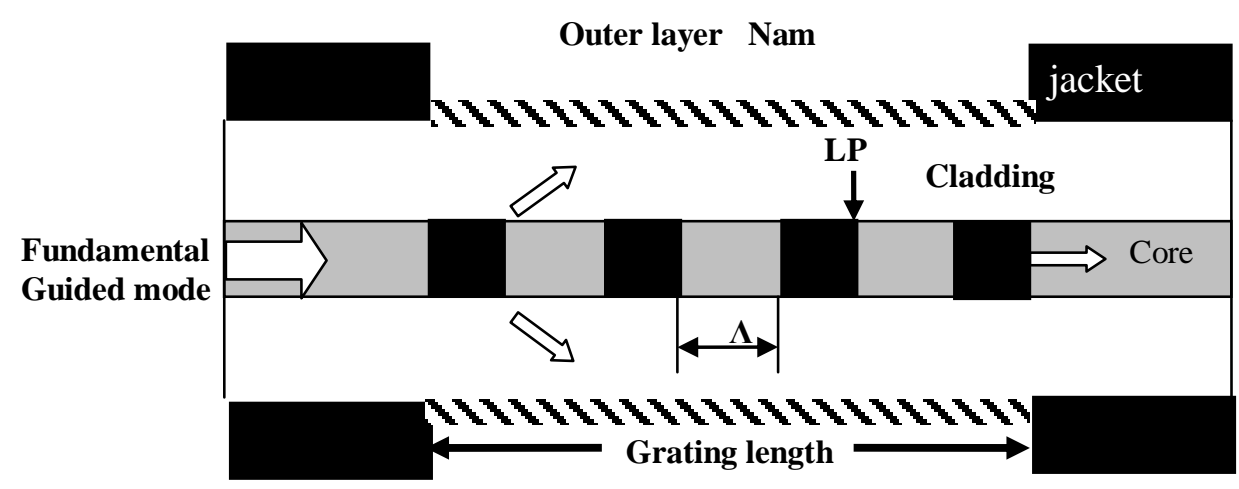

Fig.1 Long-period fiber grating 
The excited cladding mode attenuates in the coated fiber part after the grating, which results in the appearance of resonance loss in the transmission spectrum.

The interaction of one mode of a fiber with other modes is commonly described with the help of coupled-mode theory in which only two modes are supposed to be nearly phasematched and capable of resonant coupling. Based on this theory, quantitative information about the coupling coefficients and spectral properties of fiber grating can be obtained $[4,5$, 6 , and 7].

\section{3-Results and discussion:}

Here, we use matlab program version 6.5 to simulate and design the transmission characteristics of an LPFG coupler[1].

$$
\begin{aligned}
& R(z)=\cos ^{2}\left(z\left(h^{2}+d^{2}\right)^{1 / 2}\right)+d^{2} \sin ^{2}\left(z\left(h^{2}+d^{2}\right)^{1 / 2}\right) /\left(h^{2}+d^{2}\right) \\
& S(z)=h^{2} \sin ^{2}\left(z\left(h^{2}+d^{2}\right)^{1 / 2}\right) /\left(h^{2}+d^{2}\right)
\end{aligned}
$$

where $R(z)$ and $S(z)$ are the normalized energies of the core and cladding modes, respectively, $\mathrm{h}$ is the coupling coefficient,$d$ is The normalized frequency.

At the exact resonance $\mathrm{d}=0$ gives a sinusoidal law of the energy exchange, showing a possibility of mutual energy transfer from one mode to another[1]:

$$
\begin{aligned}
& R(z)=\cos ^{2}(h z) \\
& S(z)=\sin ^{2}(h z)
\end{aligned}
$$

The effective indices of the cladding modes $n_{\text {eff }}^{\text {cla a }}$ are dependent on the cladding index and the index of the surrounding (outer) ambient environment $\left(n_{a m}\right)$ (see fig.1). This means that the $n$th cladding mode coupling wavelength will change as the index of the surrounding environment changes. Cladding modes are most accurately calculated using three-layer modes for case of $n_{a m}<n_{\text {eff }}^{\text {clad }}<n_{\text {clad }}$ and irradiated part within one grating period $=0.5 \mathrm{e}^{-3} \mathrm{~m}$ [1]. In this paper we focus on modeling of the leaky configuration (i.e. when $n_{\text {am }}>n_{\text {clad }}$ ).

In the two-layer model, the power attenuation coefficient of the first order cladding mode being calculated over a wide range of $n_{a m}$. Figure (3) shows that the most sensitive regions are when $n_{a m}$ is close to $n_{\text {clad }}$ and when $n_{a m}$ changes from 1.279 to 1.489 (i.e. $\Delta n \approx 0.2$ ).

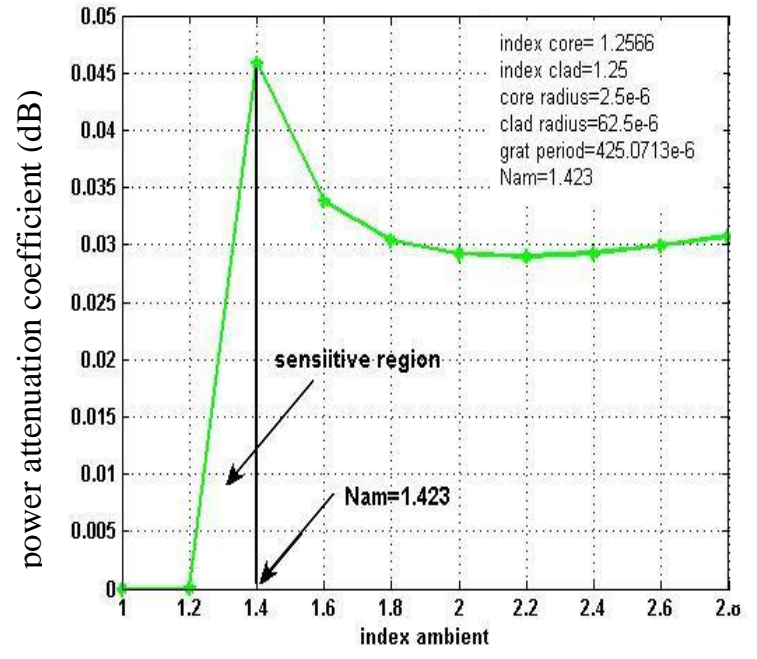

Fig.(3) Effect the surrounding medium

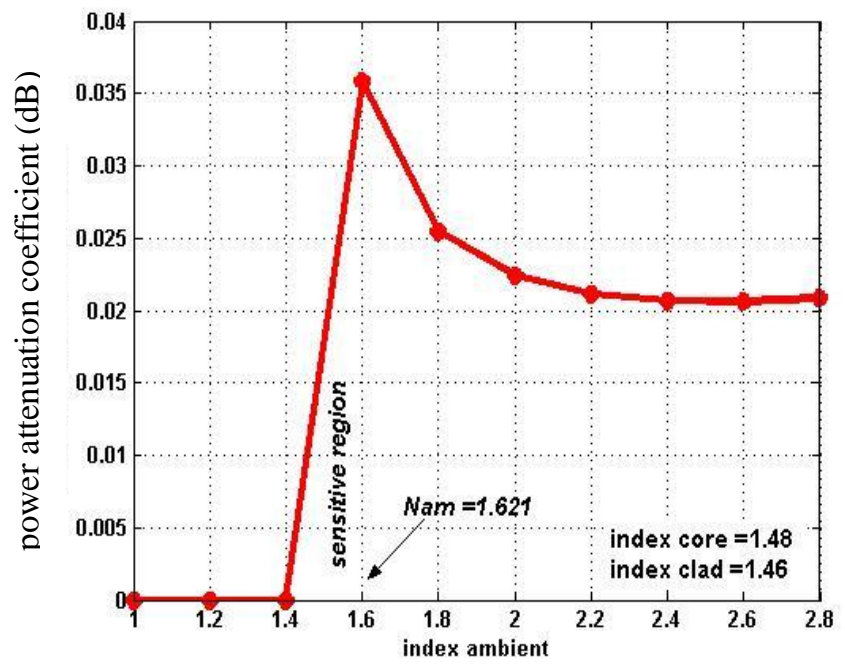

Fig.(4) Effect the surrounding medium 
The power attenuation coefficient shift shows a dramatic change from a sharp increase of $0.00 \mathrm{~dB}$ to a sharp of $0.03788 \mathrm{~dB}$. This "switching property ", in this sensitive region, has a potential application in optical communications and optical sensors(i.e. work region of our LPFG model will be only in sensitive region).

Fig.(4) shows the same effect result but with change to the core refractive index, cladding refractive index and surrounding refractive index $\left(n_{a m}>n_{\text {clad }}\right)$.From the equation (5)[1], show the relationship between input signal wavelength and index ambient :-

$\lambda_{\text {wavelength }}=A \pi L_{L P G} N_{\text {am }} N_{\text {clad }}$

Where $A$ is fiber core area(1-10) $\mu \mathrm{m}^{2}$. From the above equation we can design our the LPFG modeling in order to be used as a sensor. Our model consists of index ambient (1.4 to 1.48) range from, that is larger than cladding index (i.e. $\Delta n \approx 0.2$ ). Figs $(5,6,7,8)$ show our model, where this figs show effective index ambient $\left(N_{a m b}^{\text {eff }}\right)$ on power attenuation coefficient, we notice a dramatic change and sensitive region and power attenuation coefficient value at $N_{a m h}^{e f f} \approx 1.61$.

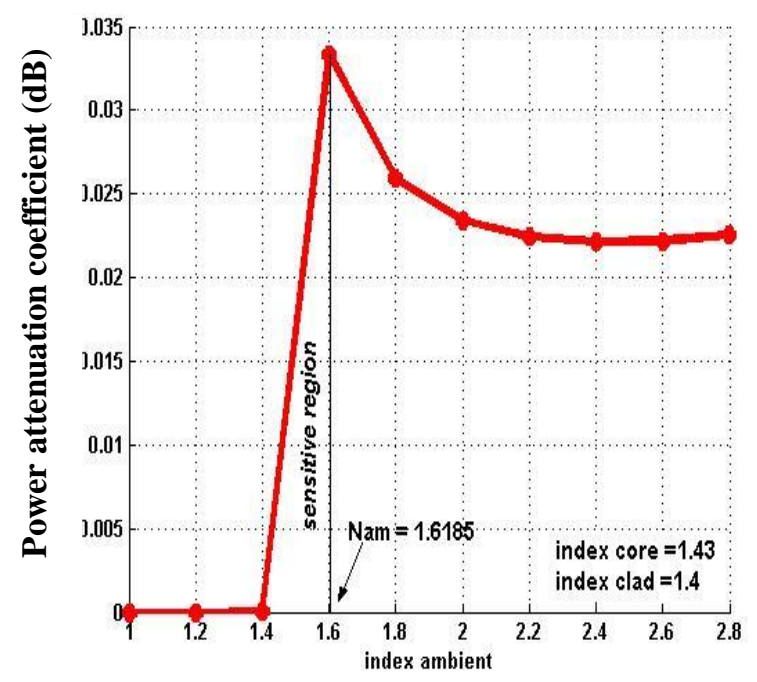

Fig.(5) Modeling index ambient

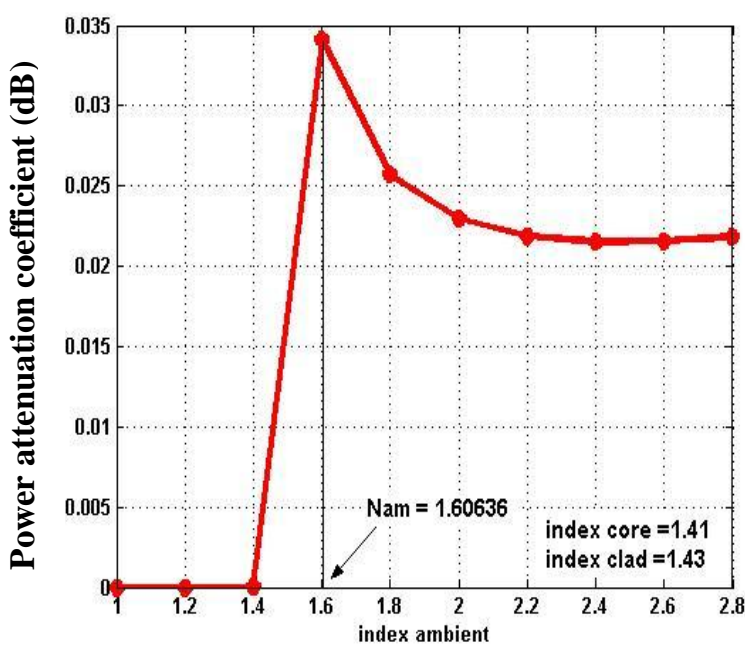

Fig.(7) Modeling index ambient

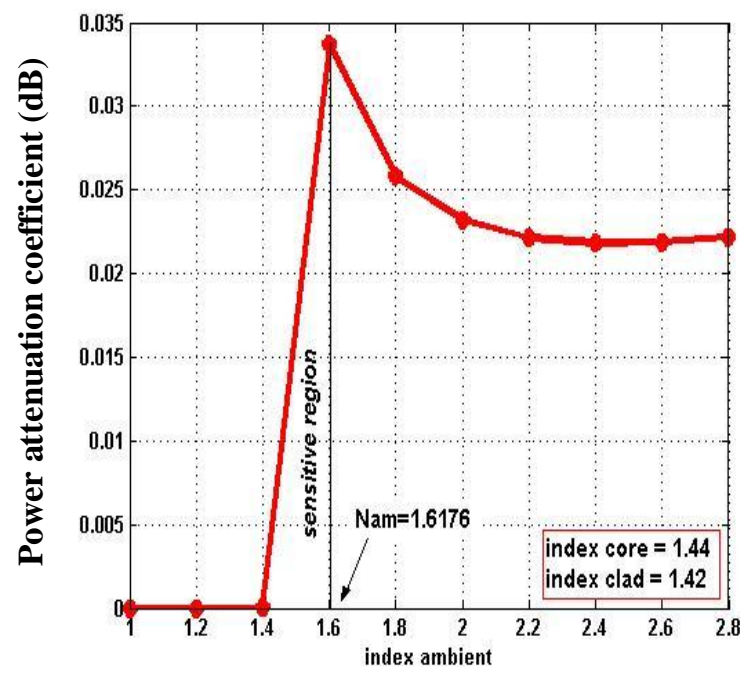

Fig.(6) Modeling index ambient

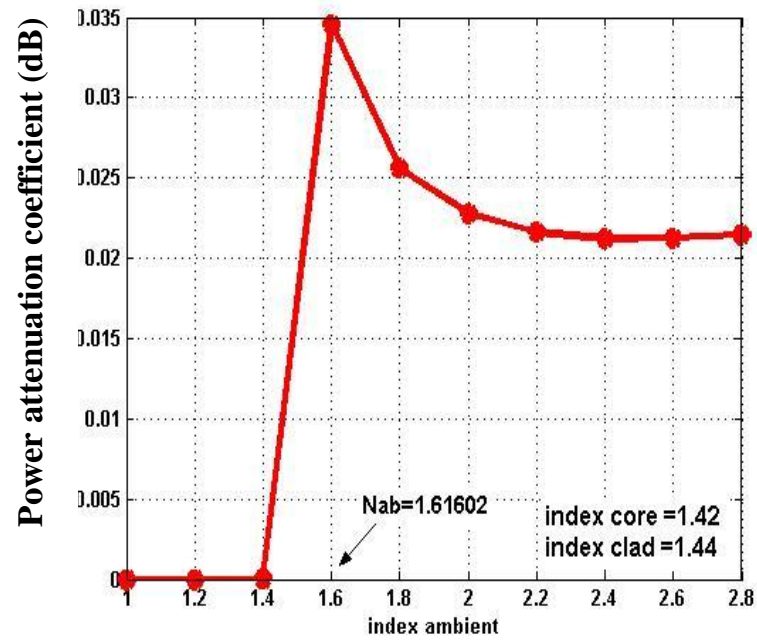

Fig.(8) Modeling index ambient 
Table (1) Wavelength used in modeling

\begin{tabular}{|l|l|l|l|l|}
\hline$\lambda_{\text {eff }}(\mathrm{nm})$ & $N_{\text {clad }}$ & $N_{\text {amb }}$ rang & $N_{\text {amb }}^{\text {eff }}>N_{\text {clad }}$ & $\Delta n \cong$ \\
\hline 1523 & 1.4 & $1.4213-1.61852$ & 1.618524689 & 0.2185246 \\
\hline 1533 & 1.42 & $1.4365-1.61767$ & 1.617679101 & 0.1976791 \\
\hline 1543 & 1.43 & $1.4472-1.60636$ & 1.606366659 & 0.1763666 \\
\hline 1553 & 1.44 & $1.4568-1.61602$ & 1.616022964 & 0.1760229 \\
\hline
\end{tabular}

From the figs and the table above notice the difference between cladding refractive index and surrounding refractive index must be $\Delta n \approx 0.2$, i.e. working region will be in sensitive region ( $N_{a m b}^{\text {eff }}>N_{\text {clad }}$ ).we will use our model as a sensor(shift transmission spectrum profile).

Figs. $(9,11,13)$ illustrate the input signal power of LPFG.

Figs. $(10,12,14)$ present the shift process for transmission spectrum (output signal power of LPFG).

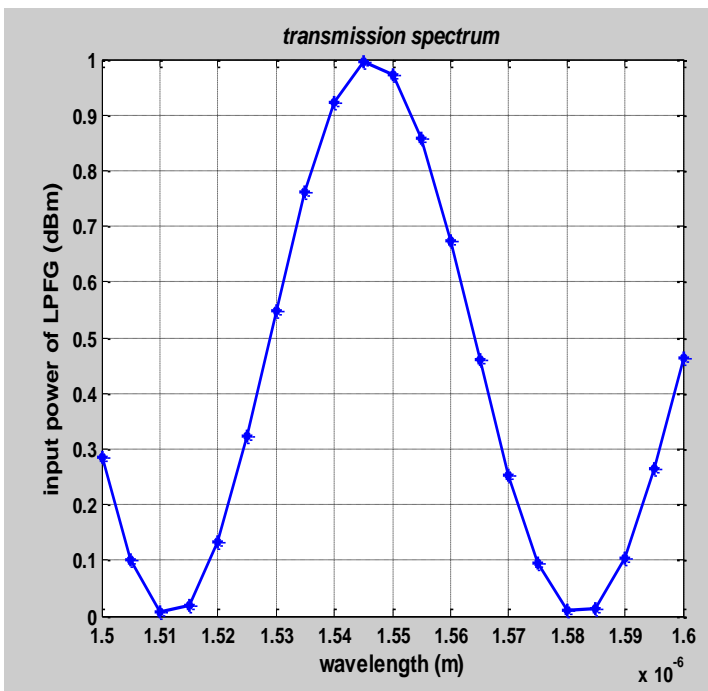

Fig.(9) transmission spectrum

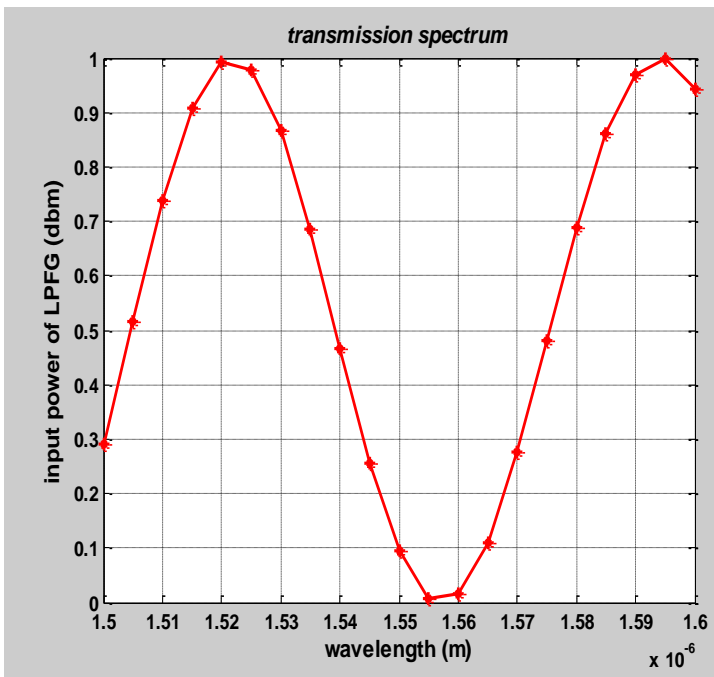

Fig.(11) transmission spectrum

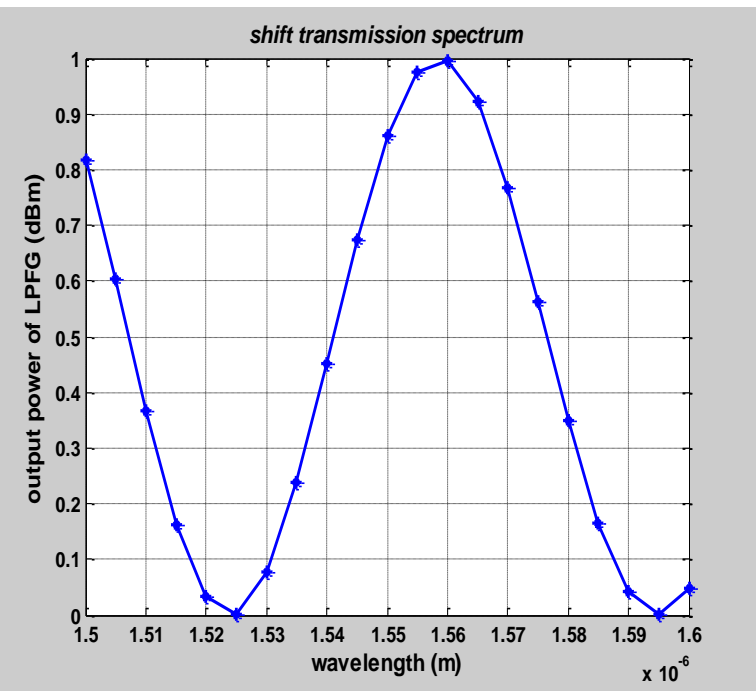

Fig.(10) shift transmission spectrum

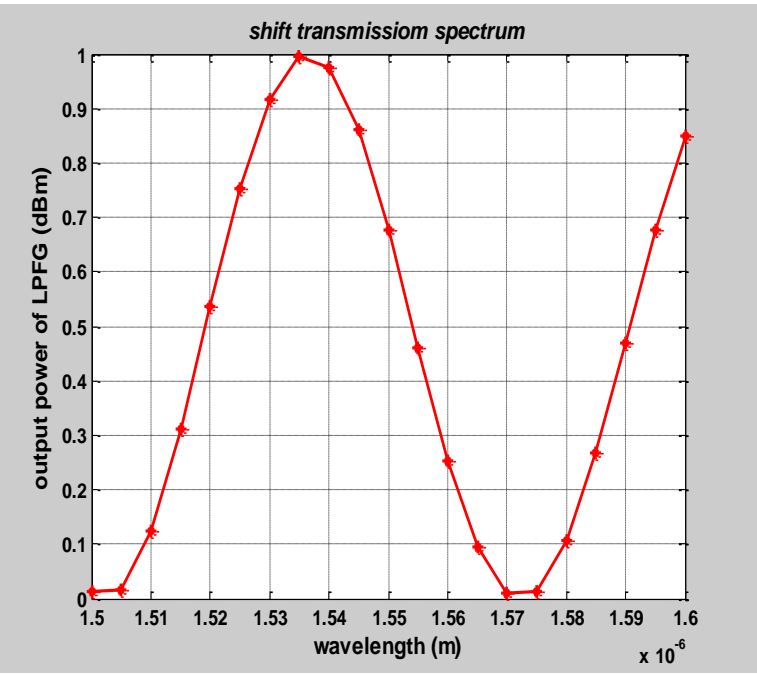

Fig.(12) shift transmission spectrum 


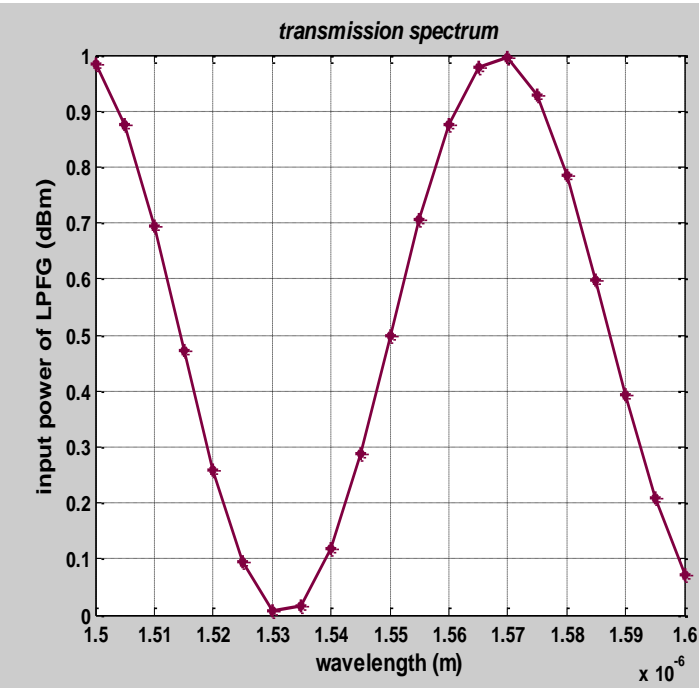

Fig.(13) transmission spectrum

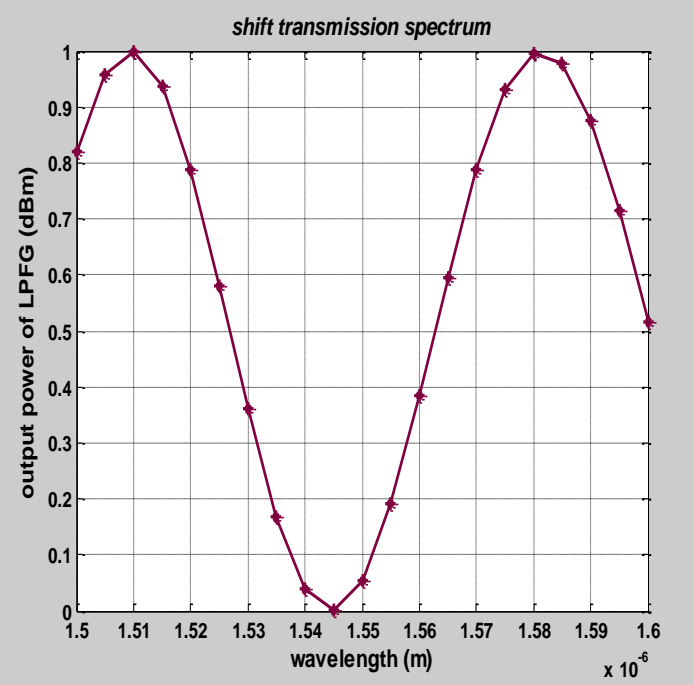

Fig.(14) shift transmission spectrum

From figs above, we notice shift to transmission spectrum profile (input signal of LPFG). This shifting depends on many parameters $\left(\Lambda_{L P G}, N_{a m}, N_{\text {clad }}, \ldots . ..\right)$. In our modeling we focused on surrounding refractive index, to take any point from transmission spectrum we notice the shift value to the wavelength $\approx 15 \mathrm{~nm}$ that equal $10 * N_{\text {amb }}^{\text {eff }} \approx 16.1$. (example: figs. $(9,10)$ the value at the wavelength $1510 \mathrm{~nm}$ in input signal become the same value at shift wavelength $1525 \mathrm{~nm}$ in output signal that shift wavelength equal $\approx 15 \mathrm{~nm}$ ). Therefore, we will control on the shift wavelength to the input signal LPFG (rang fiber optical 1500nm to $1600 \mathrm{~nm}$ ) through the surrounding refractive index, in the other word LPFG is working as sensor.

The table (2) shows some examples in shift wavelength:

Table (2) shift Wavelength.

\begin{tabular}{|l|l|l|l|l|}
\hline $\begin{array}{l}\text { Input } \\
\text { wavelength }\end{array}$ & $N_{\text {amb }}^{\text {eff }}>N_{\text {clad }}$ & $\begin{array}{l}\text { Output shift } \\
\text { wavelength }\end{array}$ & $N_{\text {clad }}, N_{\text {core }}$ & Different wavelength \\
\hline $1510 \mathrm{~nm}$ & 1.618524 & $1525 \mathrm{~nm}$ & $1.4,1.43$ & $1526-1512=15 \mathrm{~nm}$ \\
\hline $1555 \mathrm{~nm}$ & 1.617679 & $1570 \mathrm{~nm}$ & $1.42,1.44$ & $1570-1555=15 \mathrm{~nm}$ \\
\hline $1530 \mathrm{~nm}$ & 1.606366 & $1545 \mathrm{~nm}$ & $1.43,1.41$ & $1545-1530=15 \mathrm{~nm}$ \\
\hline
\end{tabular}

We take another example in shift transmission spectrum through taking another model from effective surrounding refractive index to power attenuation coefficient. Figs. $(15,16)$ show effective index ambient on power attenuation coefficient, we notice a dramatic change and sensitive region and power attenuation coefficient value at $N_{a m b}^{\text {eff }} \approx 1.44$.

From the figs. $(15,16)$ and the table(3) notice the difference between cladding refractive index and surrounding refractive index must be $\Delta n \approx 0.2$, i.e. working region will be in sensitive region $\left(N_{\text {amb }}^{\text {eff }}>N_{\text {clad }}\right)$. we will use another model as a sensor (shift transmission spectrum profile). 


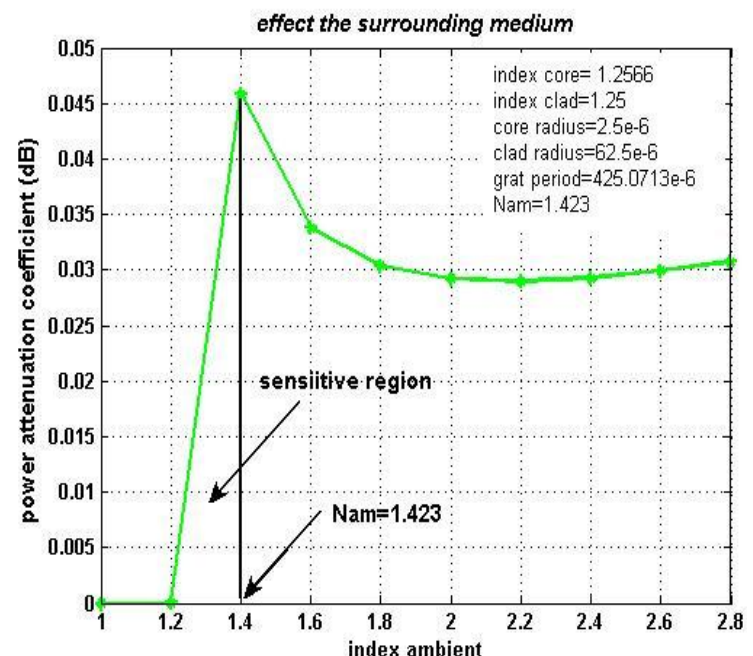

Fig.(15) Modeling index ambient

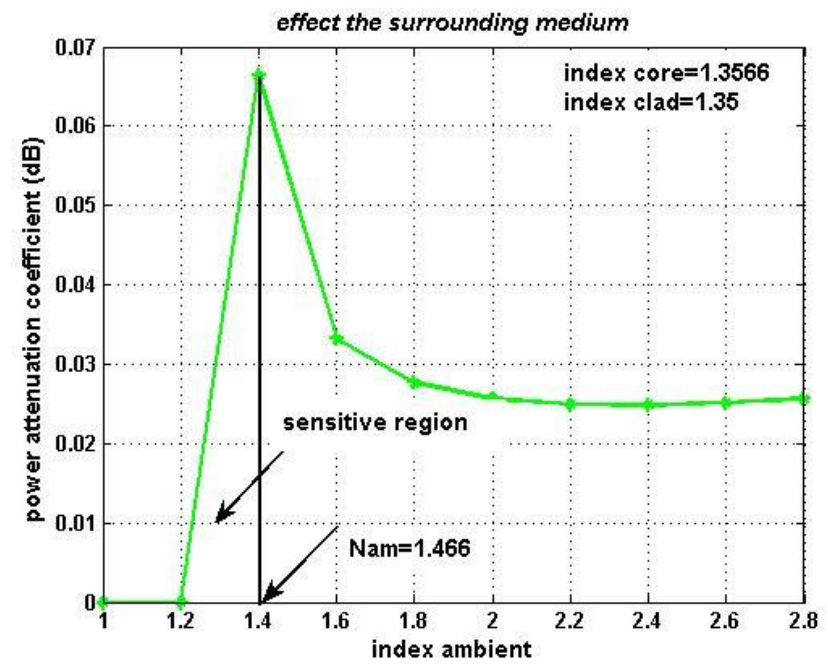

Fig.(16) Modeling index ambient

Table (3) Wavelength used in another modeling

\begin{tabular}{|l|l|l|l|l|}
\hline$\lambda_{\text {eff }}(\mathrm{nm})$ & $N_{\text {clad }}$ & $N_{\text {amb }}$ rang & $N_{\text {amb }}^{\text {eff }}>N_{\text {clad }}$ & $\Delta n \cong$ \\
\hline 1520 & 1.25 & $1.2113-1.423$ & 1.42311011 & 0.211810 \\
\hline 1545 & 1.35 & $1.2136-1.466$ & 1.46632101 & 0.232721 \\
\hline
\end{tabular}

Figs. $(17,19)$ illustrate the input signal power of LPFG.

Figs. $(18,20)$ presents the shift process for transmission spectrum profile(output signal power of LPFG).

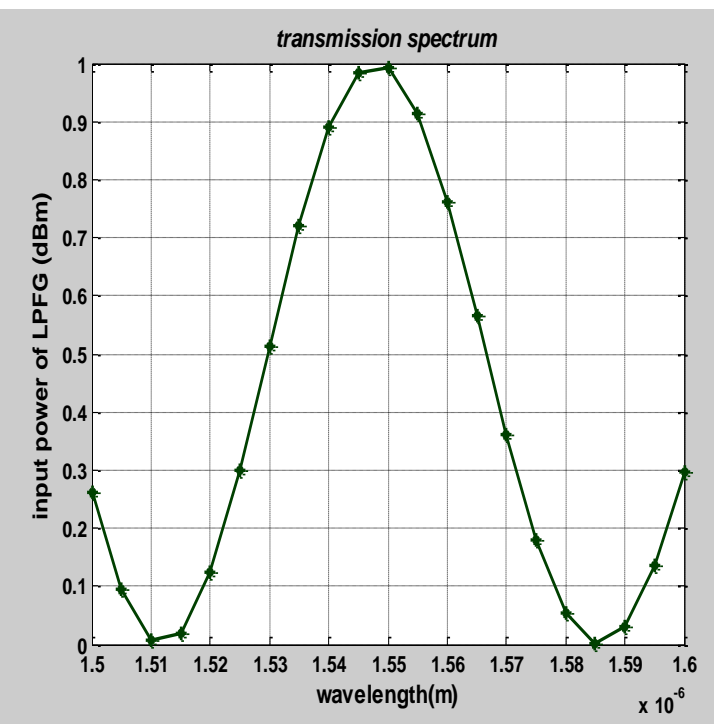

Fig.(17) transmission spectrum

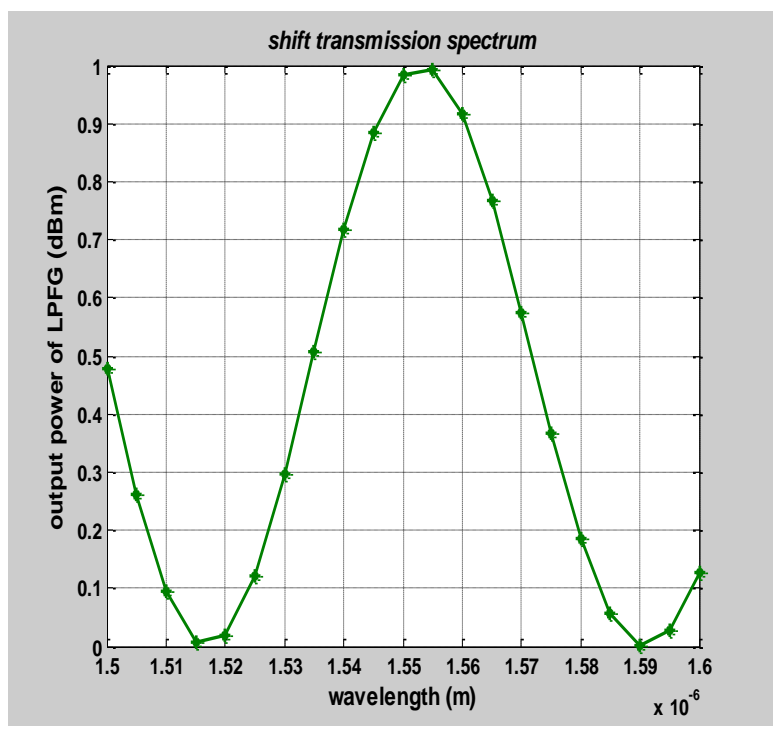

Fig.(18) shift transmission spectrum 


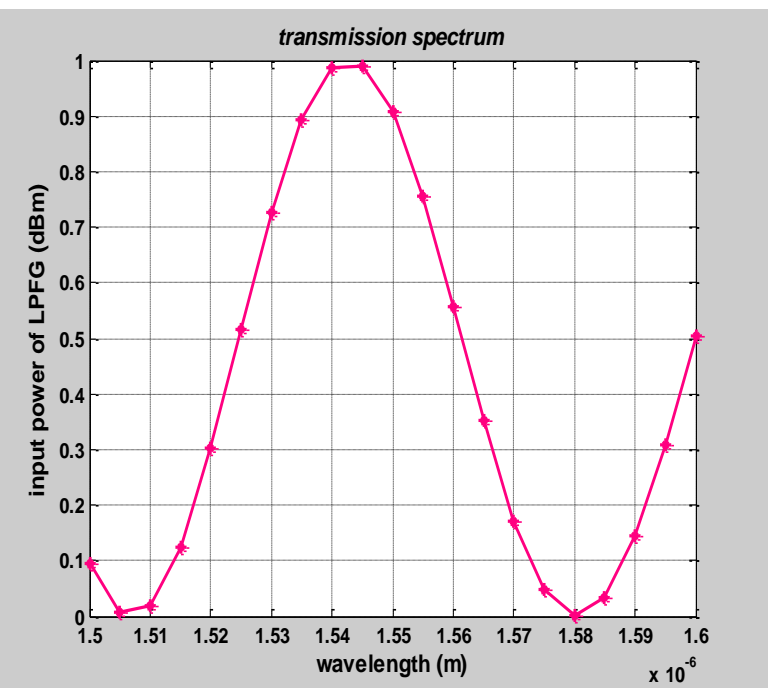

Fig.(19) transmission spectrum

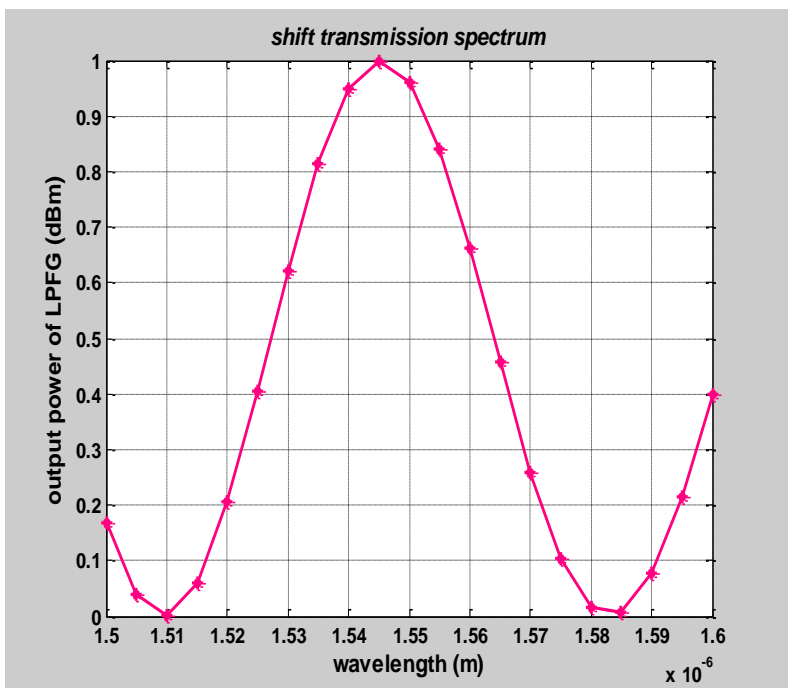

Fig.(20) shift transmission spectrum

From figs. above, we notice shift to transmission spectrum profile (input signal of LPFG). This shifting depends on many parameters $\left(\Lambda_{L P G}, N_{a m}, N_{\text {clad }}, \ldots . ..\right)$. In another modeling we notice the shift value to the wavelength $\approx 5 \mathrm{~nm}$ that equal $3.5^{*} N_{a m b}^{\text {eff }} \approx 5.04$. (example: figs. $(17,19)$ the value at the wavelength $1585 \mathrm{~nm}$ in input signal become the same value at shift wavelength $1590 \mathrm{~nm}$ in output signal that shift wavelength equal $\approx 5 \mathrm{~nm}$ ). Therefore, we will control on the shift wavelength to the input signal LPFG through the surrounding refractive index, in the other word LPFG its working as sensor.

The table(4) show some the example in shift wavelength:

Table (4) shift Wavelength.

\begin{tabular}{|l|l|l|l|l|}
\hline $\begin{array}{l}\text { Input } \\
\text { wavelength }\end{array}$ & $N_{\text {amb }}^{\text {eff }}>N_{\text {clad }}$ & $\begin{array}{l}\text { Output shift } \\
\text { wavelength }\end{array}$ & $N_{\text {clad }}, N_{\text {core }}$ & Different wavelength \\
\hline $1585 \mathrm{~nm}$ & 1.423 & $1590 \mathrm{~nm}$ & $1.25,1.2566$ & $1590-1585=5 \mathrm{~nm}$ \\
\hline $1580 \mathrm{~nm}$ & 1.466 & $1585 \mathrm{~nm}$ & $1.35,1.3566$ & $1585-1580=5 \mathrm{~nm}$ \\
\hline
\end{tabular}

\section{4- Conclusions:}

In this paper, We have presented the LPFG model by changing the ambient refractive index higher than cladding refractive index $\left(n_{a m}>n_{\text {clad }}\right)$. The results may come up with many points:

1. The first model (figs.5, 6, 7, 8) works over a wide range (1.4-1.6).i.e. many inputs at the same time.

2. The second model (figs 15,16$)$ works over a wide range (1.2-1.4).i.e. many inputs at the same time.

3. is presented effective ambient index to LPFG working where the difference between cladding refractive index and surrounding refractive index in our two modeling must be equal to $\Delta n \approx 0.2$.

4. In first modeling, the best working point, is when power attenuation coefficient is of a higher value $\approx 0.03788 \mathrm{~dB}$. 
5. In second modeling, the best working point, is when power attenuation coefficient is of a higher value $\approx 0.04675 \mathrm{~dB}$ and $\approx 0.06875 \mathrm{~dB}$.

6- LPFG its working as sensor, therefor the input signal to this device is shifted, depending on the value of surrounding refractive index. In our two modeling shift values of the wavelength are equal $10^{*} N_{a m b}^{\text {eff }} \approx 16.1$ and $3.5^{*} N_{a m b}^{\text {eff }} \approx 5.04$.

7- In two our modeling notice power attenuation coefficient dependent on core index and clad index not only on surrounding refractive index, therefore, the shift value to input signal depends on this parameters, any change to this parameters causes change in the shift value of LPFG input signal wavelength.

\section{5-References:}

1- Furat Y. Abdulrazak "the effect of ambient refractive index on the action of Long Period Fiber Grating", Dept. of software Eng. University of Mosul, Iraq.

2- Rosane Falate, Jose Luis Fabris, Marcia Muller and Hypolito Jose Kalinowski. " Long Period Grating Sensor to monitor Fuel Quality". Asian Journal of Physics. Av. Sete de Setembro, 3165-80230-901. Curitiba,Brazil 2002.

3- Jinho Bae, Jun Kye Base,Joohwan Chun, " Analysis for long period fiber gratings using thermal kernel function", Optical Society of America 2004.

4- Youngjoo Chung, Un-Chul Paok "Fabrication and Performance Characteristics of Optical Fiber Gratings for Sensing Applications", Dept. of Info. And Comm., Kwangju Institute of Science and Technology, Kwnagju 500-712,Korea.

5- Gerd Keiser," Optical Fiber Communications", McGraw-Hill Higher Education, International Editions 2000,www.mhhe.com.

6-A.M. Vengsarkar, P.J.Lemaire," Long-period grating theory",Glass photosensitivity and Fiber Gratings, Fiber Research Center GPI RAN.

7-Kin Seng Chiang, "Analysis of Two Parallel Long-Period Fiber Gratings", Journal of Lightwave Technology, VOL.22,NO.5, MAY 2004. 